\title{
Utilização da tecnologia CAD/CAM em clínicas odontológicas: revisão de literatura
}

\author{
Use of CAD/CAM technology in dental clinics: literature review
}

Uso de tecnología CAD/CAM en clínicas dentales: revisión de la literatura

Flávio Augusto de Moraes Palma ${ }^{1 *}$, Letícia Martim¹, João Vitor Oliveira de Amorim¹, Gustavo Baruc Andrade Abreu ${ }^{1}$, Gustavo Henrique Santos de Almeida ${ }^{1}$, Victor Arthur Rodrigues de Souza ${ }^{1}$, Marcus Vinícius Simões Feitosa ${ }^{1}$, Ezequiel Santos Barbosa1, Izabela Lima Góis ${ }^{1}$, Flavia Pardo Salata Nahsan².

\section{RESUMO}

Objetivo: Identificar a aplicabilidade do uso do CAD/CAM em clínicas odontológicas, assim como os benefícios da utilização. Métodos: trata de estudo é uma revisão de literatura de pesquisa qualitativa, onde realizou-se quatro etapas onde as mesmas foram divididas das seguintes formas: (1) Seleção das palavras chaves da pesquisa; (2) Busca de artigos em bases de dados e Portais eletrônicos; (3) Selecionar estudos com base em critério de inclusão/exclusão; (4) Leitura dos artigos na integra. Resultados: de acordo a pesquisa, foram selecionados 21 artigos, divididos por autores, título, ano e periódicos. Diante dos artigos encontrados, os autores relatam a eficiência clínica, pois o sistema CAD/CAM pode atuar em diversos procedimentos clínicos, obtendo um ótimo resultado no tempo de trabalho, qualidade no procedimento e satisfação do paciente, por ser um procedimento rápido e menos invasivo. Considerações finais: Considera que os tratamentos propostos podem variar, desde a produção de coroas unitárias, facetas, prótese sobre implante, implantes dentários e até a realização de guias cirúrgicos e benéficos gerados pelo CAD/CAM podem ser: ganho de eficiência e tempo de trabalho; pode aumentar a qualidade e precisão do seu trabalho e o tempo de espera do paciente ao seu tratamento.

Palavras-chave: Odontologia, Tecnologia odontológica, Reabilitação bucal.

\begin{abstract}
Objective: To identify the applicability of using CAD/CAM in dental clinics, as well as the benefits of using it. Methods: treats of study is a qualitative research review literature, in which four stages were carried out, where they were divided into the following ways: (1) Selection of the research keywords; (2) Search for articles in databases and electronic portals; (3) Select studies based on inclusion / exclusion criteria; (4) Reading of the articles in full. Results: according to the research, 21 articles were selected, divided by authors, title, year and journals. In view of the articles found, the authors report the clinical efficiency, since the CAD/CAM system can act in several clinical procedures, obtaining an excellent result in working time, quality in the procedure and patient satisfaction, as it is a quick and less invasive procedure. Final considerations: Considers that the proposed treatments can vary, from the production of single crowns, veneers, implant prosthesis, dental implants and even the realization of surgical and beneficial guides generated by CAD/CAM can be: efficiency gain and working time; it can increase the quality and precision of your work and the patient's waiting time for your treatment.
\end{abstract}

Key words: Dentistry, Dental technology, Oral rehabilitation.

\footnotetext{
1 Universidade Federal de Sergipe, Lagarto - SE. *E-mail: flavioampodonto@gmail.com

2 Universidade Federal de Sergipe, Aracaju - SE.
}

SUBMETIDO EM: 1/2021

ACEITO EM: 1/2021

PUBLICADO EM: 2/2021 


\section{RESUMEN}

Objetivo: Identificar la aplicabilidad del uso de CAD/CAM en clínicas dentales, así como los beneficios de su uso. Métodos: el estudio viene es una revisión de la literatura de investigación cualitativa, donde se realizaron cuatro etapas, donde se dividieron de las siguientes formas: (1) Selección de las palabras clave de investigación; (2) Búsqueda de artículos en bases de datos y portales electrónicos; (3) Seleccionar estudios basados en criterios de inclusión / exclusión; (4) Lectura íntegra de los artículos. Resultados: según la investigación, se seleccionaron 21 artículos, divididos por autores, título, año y revistas. A la vista de los artículos encontrados, los autores reportan la eficiencia clínica, ya que el sistema CAD/CAM puede actuar en varios procedimientos clínicos, obteniendo un excelente resultado en tiempo de trabajo, calidad en el procedimiento y satisfacción del paciente, por ser un procedimiento rápido y menos invasivo. Consideraciones finales: Considera que los tratamientos propuestos pueden variar, desde la producción de coronas unitarias, carillas, prótesis de implantes, implantes dentales e incluso la realización de guías quirúrgicas y beneficiosas generadas por CAD/CAM pueden ser: ganancias de eficiencia y tiempo de trabajo; puede aumentar la calidad y precisión de su trabajo y el tiempo de espera del paciente para su tratamiento.

Palabras clave: Odontología, Tecnología dental, Rehabilitación oral.

\section{INTRODUÇÃO}

Atualmente, milhares de cirurgiões dentistas espalhados pelo mundo trabalham de forma tradicional com relação a fabricação de próteses totais, coroas fixas, facetas, entre outros, e quando falamos em forma de trabalho tradicional destes procedimentos, o processo tradicional de fabricação de coroas inclui: preparação do dente, moldagem, extração do dente tratado, montagem do conjunto de mordedura, modelagem em cera, fundição e acabamento centrífugo de revestimento e sinterização de porcelana ou polimerização de resina. Sendo que o processo tradicional de fabricação de próteses envolve muito trabalho manual de dentistas e técnicos dentais sendo que todas essas etapas dependem significativamente da habilidade do técnico de prótese dentária (ROMERO L, et al., 2015).

A tecnologia recém moderna de design auxiliado por computador e fabricação assistida por computador Computer Aided Design (CAD) / Computer Aided Manufacturing (CAM) tem estado cada vez mais nos consultórios odontológicos espalhados pelo mundo, melhorado significativamente o tempo de trabalho e a qualidade dos procedimentos odontológicos (GAN N, et al., 2019). O mesmo surgiu por volta dos anos 80 , na Universidade de Zurich, Suíça, quando o Professor Werner H. Mörmann desenvolveu o primeiro sistema CAD/CAM odontológico, no qual era possível a fabricação de inlays a partir de um bloco cerâmico, porem em 1973, Duret, através dos seus desenvolvimentos, ele realizou o primeiro tratamento restaurador dentário usando habilidades digitais na implantodontia (PYO SW, et al., 2020).

Esse sistema permite que o cirurgião dentista faça uma espécie de scanner na boca de seu paciente, sem o uso de material de moldagem, além de criar a prótese rapidamente por meio de uma fresadora. A tecnologia CAD/CAM é uma tecnologia que oferece uma manufatura padronizada no processo de criação, que permite um tempo reduzido e o custo eficaz na fabricação de dentes e restaurações suportadas por implantes. Os desenvolvimentos nas técnicas CAD/CAM tornaram possível projetar e fabricar digitalmente próteses totais usando técnicas de fresamento e diante desta tecnologia inovadora, foi possível realizar restaurações indiretas em consultório totalmente gerenciadas pelo clínico, podendo ter como as vantagens: menor custo para o paciente, execução mais rápida das restaurações e a exclusão da fase provisória (HASSAN B, et al., 2017a).

Com o aumento de melhorias em tecnologias e materiais clínicos, o uso e o desenvolvimento de materiais restauradores da cor do dente têm aumentado em conjunto com a tecnologia CAD / CAM e as demandas estéticas dos pacientes, notadamente no campo de blocos de resina composta, e os novos materiais cerâmicos em combinação com a cimentação adesiva fornecem um fluxo de trabalho confiável, previsível e econômico, sendo possível fabricar várias peças em restaurações ultrafinas, sendo elas minimamente 
invasivas ou mesmo não invasivas podendo ser em uma única consulta (DEL CURTO F, et al., 2108; SELZ CF, et al., 2016; LEE JH, et al., 2019a).

O sistema CAD/CAM é amplamente utilizado, uma vez que a impressão digital é mais confortável para os pacientes (principalmente a população idosa vulnerável ou mais jovens), o acaba se tornando um diferencial competitivo (BARENGHI L, et al., 2019).

Acompanhando o CAD/CAM, o escaneamento facial digital, que é uma tecnologia em rápida evolução com uma ampla gama de aplicações nas áreas de engenharia biomédica e design industrial que permite capturar impressões das arcadas dentárias de pacientes sem ter que usar materiais de impressão convencionais, mas usando apenas uma fonte de luz (uma grade de luz estruturada ou, mais raramente, um laser) que é projetado nos dentes a serem digitalizados (MANGANO F, et al., 2019).

Os avanços na tecnologia de imagem tridimensional (3D) e scanners digitais permitiram uma melhor visualização das estruturas anatômicas e maior precisão e exatidão para a colocação do implante (LEE JD, et al., 2019b). Assim como os scanners intraorais, também existem articuladores digitais, que por sua vez permitem a correção cinemática de próteses dentárias estaticamente projetadas e restaurações dentárias usando sistemas CAD/ CAM (SOLABERRIETA E, et. al., 2015).

Existem diversas tecnologias disponíveis em um fluxo de trabalho restaurador digital que facilitam a estabilização de questões funcionais e estéticas complexas. $\mathrm{Na}$ verdade, os atuais sistemas CAD/CAM odontológicos (por exemplo, CEREC AC Omnicam ou CEREC Primescan AC, Sirona Dental Systems, Charlotte, NC) e blocos compostos fresáveis (por exemplo, Lava Ultimate e Paradigm MZ100 da 3M ESPE; Telio e Tetric CAD da Ivoclar Vivadent; CeraSmart por GC; Enamic por VITA) permitem que os dentistas entreguem próteses provisórias previsíveis em uma consulta (LESAGE BP, 2020).

Existem outras formas de se obter o sorriso do paciente como Digital Smile Design (DSD - Digital Smile Design) que é um software idealizado pelo Dr. Christian Coachman que permite diagnosticar, desenhar e comunicar ao cirurgião dentista e ao paciente o resultado final desejado através da reabilitação oral (HAENGGI R, et al., 2017).

O sistema CAD/CAM em consultórios odontológicos realmente se torna uma ferramenta que irá trazer ao cirurgião dentista benefícios, e ir além dos procedimentos clínicos em seus atendimentos? Diante dos fatos, as hipóteses aos seus benefícios do CAD/CAM, podem se relacionar principalmente ao tempo nos procedimentos, eficiência, e comodidade ao paciente. Este trabalho é importante para compreendermos como o CAD/CAM se torna uma ferramenta de trabalho diferenciada no dia a dia do cirurgião dentista, que faz presente nos dias atuais nas clínicas odontológicas e tem como objetivo principal, identificar a aplicabilidade do uso do CAD/CAM em clínicas odontológicas, assim como os benefícios da sua utilização.

\section{MÉTODOS}

Esta revisão de literatura integrativa trata-se de uma pesquisa qualitativa, que consistiu das seguintes etapas: (1) Seleção das palavras chaves da pesquisa; (2) Busca de artigos em bases de dados e Portais eletrônicos; (3) Selecionar estudos com base em critério de inclusão/exclusão; (4) Leitura dos artigos na integra. Na primeira etapa, realizou-se uma busca no Decs descritores, com três palavras: odontologia, odontologia tecnológica, reabilitação oral. A segunda etapa constituiu-se pela busca de artigos nos portais eletrônicos PUBMED E BVS, e das bases de dados MEDLINE E LILACS. Na terceira etapa, os artigos encontrados para a pesquisa somaram um total de 1819, e logo após os critérios de inclusão/exclusão, restando 21 artigos para a pesquisa. Na quarta etapa os artigos foram lidos na integra.

Os critérios de inclusão/exclusão foram realizados de acordo a cada portal periódico e base de dados, distribuídos da seguinte forma: no portal de dados BVS, foram aplicadas as palavras chaves encontradas no Decs descritores, obtendo um total de 47 artigos, no qual foi utilizado como critérios de exclusão, o período de artigos recentes de 5 anos como seleção, obtendo um total de 17 artigos, e logo após foi utilizado como como critérios de exclusão o filtro banco de dados MEDLINE, obtendo 12 artigos, que logo após utilizar o critério de inclusão da leitura do resumo, foram selecionados 5 artigos. 
Os critérios de inclusão/exclusão para a base de dados Lilacs foram realizados da seguinte forma: pelo portal de dados BVS, foram aplicadas as palavras chaves encontradas no Decs descritores, obtendo um total de 47 artigos, no qual foi utilizado como critérios de exclusão o período de artigos recentes de 5 anos como seleção, obtendo um total de 17 artigos, e logo após foi utilizado como critérios de exclusão filtro o banco de dados LILACS, no qual obteve 2 artigos que foram incluídos.

No Pubmed, para critérios de inclusão/exclusão foram aplicadas as palavras chaves encontradas no Decs descritores, obtendo um total de 954, e logo em seguida foi adicionado como critérios de exclusão um filtro de artigos recentes de 5 anos como seleção, no qual resultou em 176 artigos. Posteriormente, como critérios de exclusão foi utilizado o filtro de texto completo, onde resultou em um total de 136 artigos que logo após foi utilizado o filtro de artigos de revisão da literatura para critério de exclusão, obtendo 14 artigos, que logo após utilizar o critério de inclusão leitura do resumo, restaram 2 artigos para a pesquisa.

No Portal Periódico Capes, para critérios de inclusão/exclusão foram aplicadas as palavras chave encontradas no Decs descritores, obtendo um total de 818, e logo em seguida, para critério de exclusão foi adicionado um filtro de artigos recentes de 5 anos como seleção, no qual resultou em 434 artigos. Posteriormente ao filtro de tempo do artigo, foi utilizado como critérios de exclusão o filtro computer aided design, onde resultou em um total de 44 que logo após utilizar o critério de inclusão leitura do resumo desses artigos, foram selecionados 12 artigos para a pesquisa. Logo em seguida a seleção dos artigos, os mesmos foram lidos na integra, completando assim a quarta etapa.

\section{RESULTADOS E DISCUSSÃO}

Os artigos encontrados nas bases de dados e portais periódicos, somaram um total de 1819 artigos. Os resultados dos artigos, logo passarem pelos critérios de inclusão/exclusão, somaram um total de 21 artigos, onde os mesmos foram lidos na integra, e sendo divididos pelos autores principais, título, ano e plataforma utilizada. Sendo assim os resultados foram separados de acordo ao banco de dados/portais periódicos (Quadro1). 
Quadro 1 - Seleção dos artigos selecionados, divididos pelos autores principais, título, ano, e plataforma utilizada.

\begin{tabular}{|c|c|c|c|}
\hline Autores & Título & Ano publicação & Plataforma \\
\hline Sun YC, et al. (2018) & $\begin{array}{l}\text { Pesquisa e desenvolvimento de design digital e fabricação de próteses } \\
\text { totais. }\end{array}$ & 2018 & Pubmed \\
\hline LeSage BP (2020) & $\begin{array}{c}\text { CAD / CAM: Aplicações para ligação transicional para restaurar a } \\
\text { dimensão vertical oclusal. }\end{array}$ & 2020 & Pubmed \\
\hline Saratti CM, et al. (2020) & $\begin{array}{c}\text { Reabilitação bucal de um caso de desgaste dentário grave: uma } \\
\text { abordagem digital, estética e funcional. }\end{array}$ & 2020 & Bvs/Medline \\
\hline Lee JD, et al. (2019) & $\begin{array}{l}\text { Fluxo de Trabalho de Tratamento Digital e Convencional Integrado na } \\
\text { Reabilitação Completa Guiada com Implante Bucal: Relato de Caso } \\
\text { Clínico. }\end{array}$ & 2019 & Bvs/Medline \\
\hline Del Curto F, et al. (2018) & $\begin{array}{c}\text { Técnica de restauração de cabeceira baseada em CAD / CAM com } \\
\text { resina composta para reabilitação adesiva de boca inteira de dentição } \\
\text { excessivamente desgastada. }\end{array}$ & 2018 & Bvs/Medline \\
\hline Hassan B, et al. (2017) & $\begin{array}{c}\text { Integração do escaneamento facial 3D em um fluxo de trabalho digital } \\
\text { para projetar CAD / CAM e fabricar próteses totais para reabilitação } \\
\text { total imediata da boca. }\end{array}$ & 2017 & Bvs/Medline \\
\hline Selz C, et al. (2016) & $\begin{array}{l}\text { Reabilitação de boca completa com materiais híbridos e totalmente } \\
\text { cerâmicos fabricados em CAD / CAM monolíticos: relato de caso e } \\
\text { acompanhamento de } 3 \text { anos. }\end{array}$ & 2016 & Bvs/Medline \\
\hline Haenggi, R, et al. (2017) & Design de sorriso em PowerPoint. & 2017 & Bvs/Lilacs \\
\hline Cuervo, JM (2018) & CAD-CAM em prótese total. Relato de caso. & 2018 & Bvs/Lilacs \\
\hline Hassan B, et al. (2017) & $\begin{array}{c}\text { Uma abordagem digital que integra o escaneamento facial em um fluxo } \\
\text { de trabalho CAD-CAM para a reabilitação de pacientes com } \\
\text { edentulismo de boca completa com implante: Um estudo clínico piloto. } \\
\text { (Relatório clínico). }\end{array}$ & 2017 & Capes \\
\hline Mehra M, et al. (2016) & $\begin{array}{l}\text { Reconstrução de enxerto fibular mandibular com tecnologia CAD / } \\
\text { CAM: um relatório clínico e revisão da literatura. }\end{array}$ & 2016 & Capes \\
\hline
\end{tabular}

REAS | Vol. 13(2) | DOI: https://doi.org/10.25248/REAS.e6370.2021 


\begin{tabular}{|c|c|c|c|}
\hline Autores & Título & Ano publicação & Plataforma \\
\hline Lee JH, et al. (2019) & $\begin{array}{c}\text { Reabilitação completa da boca contemporânea usando um desenho } \\
\text { digital do sorriso em combinação com materiais restauradores } \\
\text { convencionais e de desenho / fabricação auxiliados por computador em } \\
\text { um paciente com bruxismo. Um relato de caso. }\end{array}$ & 2019 & Capes \\
\hline Romero L, et al. (2015) & $\begin{array}{l}\text { Novo design para prototipagem rápida de modelos digitais para } \\
\text { múltiplas restaurações de implantes dentários. }\end{array}$ & 2015 & Capes \\
\hline Barenghi L, et al. (2019) & $\begin{array}{c}\text { Inovação por Design Assistido por Computador / Tecnologia de } \\
\text { Fabricação Assistida por Computador: Uma Análise da Prevenção de } \\
\text { Infecções em Ambientes Odontológicos. }\end{array}$ & 2019 & Capes \\
\hline Amornvit P e Sanohkan S (2019) & $\begin{array}{l}\text { A precisão das varreduras digitais de rosto obtidas de scanners 3D: um } \\
\text { estudo in vitro. }\end{array}$ & 2019 & Capes \\
\hline Lee $\mathrm{KH}$, et al. (2015) & $\begin{array}{l}\text { Efeitos da tecnologia de fabricação auxiliada por computador na } \\
\text { precisão de restaurações clínicas livres de metal. }\end{array}$ & 2015 & Capes \\
\hline Pyo SW, et al. (2020) & $\begin{array}{l}\text { Materiais e tecnologias cerâmicas aplicadas a obras digitais em } \\
\text { odontologia restauradora com suporte de implantes. }\end{array}$ & 2020 & Capes \\
\hline Solaberrieta, E, et al. (2015) & $\begin{array}{c}\text { Produção virtual de próteses dentárias usando um articulador virtual } \\
\text { odontológico. }\end{array}$ & 2015 & Capes \\
\hline Mangano, F, et al. (2019) & $\begin{array}{l}\text { Combinação de varreduras intraorais e faciais para o projeto e } \\
\text { fabricação de projeto assistido por computador / fabricação assistida } \\
\text { por computador (CAD / CAM) Barras suportadas por implante de } \\
\text { poliéter-éter-cetona (PEEK) para overdentures maxilares. }\end{array}$ & 2019 & Capes \\
\hline Mangano, F, et al. (2019) & $\begin{array}{c}\text { Um novo protocolo totalmente digital (SCAN-PLAN-MAKE-DONE®) } \\
\text { para o projeto e fabricação de coroas de zircônia translúcidas } \\
\text { monolíticas suportadas por implantes cimentadas em abutments } \\
\text { híbridos personalizados: um estudo clínico retrospectivo em } 25 \\
\text { pacientes. }\end{array}$ & 2019 & Capes \\
\hline Gan, N, et al. (2016) & $\begin{array}{l}\text { Precisão de impressões digitais intraorais para maxilares superiores } \\
\text { inteiros, incluindo dentições completas e tecidos moles palatinos. }\end{array}$ & 2016 & Capes \\
\hline
\end{tabular}

Fonte: Palma FAM, et al. 2021. 
O resultado dos artigos selecionados, demonstraram que há uma ampla utilização do CAD/CAM, podendo ser utilizado em diversas áreas da odontologia, em diversos procedimentos. E quando falamos de CAD/CAM, lembramos sempre de reabilitações estética, ou seja, restaurações, facetas, coroas unitárias. Sendo assim, Sarati CM, et al. (2020) em seu estudo, forneceu uma documentação passo a passo de uma reabilitação bucal, realizada com uma abordagem digital e restaurações de resina composta com o uso do CAD/CAM.

Seu uso pode não ser somente para uma única unidade dentária, mas também para arcada inteira, como demonstrou Selz C, et al. (2016) em seu estudo de relato de caso em que detalha uma abordagem CAD/CAM para a reabilitação de arcada completa de um paciente de 65 anos de idade com restaurações suportadas por dentes e implantes e fornece uma visão geral dos materiais CAD/ CAM aplicados, e do scanner intraoral. E para concretizar os estudos feitos por Selz C, et al. (2016) sobre tais matérias utilizados no CAD/CAM, Pyo SW, et al. (2020) em seu estudo, analisou a aplicação da tecnologia digital em restaurações dentárias suportadas por implantes e explora dois materiais restauradores de cerâmica utilizados globalmente: cerâmica de vidro de dissilicato de lítio e zircônia tetragonal estabilizada com vítria.

Selz C, et al. (2016) relata que com uso do CAD/CAM, as peças fresadas possuem maior estética, resistência a fratura e biocompatibilidade de acordo com o material a ser utilizado. Lee JH, et al. (2019a) em um relato de caso, utilizou um design digital do sorriso em combinação com materiais restauradores convencionais e de design / fabricação auxiliado por computador em um paciente com bruxismo, e o mesmo ressaltou que as coroas porcelanas fundidas em ouro e porcelana fundida em zircônia são ótimas para serem utilizadas em reabilitações anteriores, porem a porcelana fundida em zircônia é melhor devido a estética em relação ao paciente estudado. Já no estudo de LEE KH, et al. (2015), através de seus resultados, ele concluiu que as tecnologias digitais auxiliadas por computador podem fabricar restaurações isentas de metal que são clinicamente aceitáveis em termos de precisão.

O uso do CAD/CAM também é utilizado em reabilitações protéticas, sendo elas totais, parciais ou sobre implantes, no estudo de Sun YC, et al. (2018), o mesmo abordou os principais procedimentos clínicos da restauração de próteses totais tradicionais, elaborando a pesquisa e o status de aplicação da técnica digital em cada etapa, a fim de fornecer referência para aplicação clínica, já estudo de Lesage BP (2019), o mesmo abordou várias técnicas e terapias que foram propostas para estabelecer, determinar e restaurar a relação cêntrica ideal (RC) e a dimensão vertical da oclusão (DVO) em pacientes que necessitam de restaurações e / ou reabilitação bucal completa utilizando o CAD/CAM, comprovando que a utilização do CAD/CAM pode ser utilizada fortemente na recuperação da função mastigatória.

Lee JD, et al. (2019b) apresenta um protocolo de tratamento personalizado que envolve a retenção estratégica de dentes para servir como pilares de transição, que suportarão um guia cirúrgico de implante guiado por computador, bem como uma prótese provisória fixa. Del Curto F, et al. (2019) realizou um caso clínico, onde foi abordada uma técnica inovadora: foi inteiramente estudado e realizado em ambiente clínico, por um dentista em um computador, sem gesso ou articulador clássico, facilitando assim o tempo de trabalho. Hassan B, et al. (2017b) mostrou que a técnica proposta do CAD/CAM tem o potencial de acelerar o procedimento de reabilitação desde a prótese imediata até a prótese implantossuportada final, levando a resultados funcionais e estéticos mais previsíveis, assim como descreveu informações de escaneamento facial extra-oral com dentaduras completas com CAD/CAM para reabilitar imediatamente a dentição terminal.

Cuervo JM (2016) realizou em seu estudo, um relato de caso onde foi realizado um tratamento protético de um paciente edêntulo total superior e inferior, auxiliado por computador e tecnologia de fabricação CAD/CAM, onde o mesmo concluiu que com o auxílio do CAD/CAM, o procedimento se torna adequado, tanto nas partes de retenção, estabilidade, estética e satisfação por parte do paciente, assim como reduz o tempo de atendimento clínico para apenas uma ou duas consultas e o tempo trabalhando no laboratório. Mehra M, et al. (2016) realizou uma reabilitação completa da boca com uma prótese total maxilar e uma prótese total fixa suportada por implante mandibular onde foi confeccionada com projeto auxiliado por computador e tecnologia de usinagem auxiliada por computador. Técnica também utilizada por Lee JD, et al. (2019b) comprova que com planejamento e execução adequados, um protocolo cirúrgico de implante guiado por computador em fases e a tecnologia CAD/CAM podem trazer precisão, eficiência e previsibilidade para casos complexos de reabilitação de implantes de boca inteira. 
Romero L, et al. (2015) propôs a substituição de todos os dispositivos físicos utilizados na fabricação de próteses convencionais por meio da utilização de ferramentas digitais, como scanners 3D, softwares de desenho $C A D$, arquivos de implantes $3 \mathrm{D}$, máquinas de prototipagem rápida ou softwares de engenharia reversa, para desenvolvimento laboratorial modelos de trabalho para acabamento de revestimentos para próteses dentárias.

Amornvit P e Sanohkan S (2019) em seus estudos, os mesmos avaliaram a qualidade de imagem de quatro equipamentos de scanners faciais, sendo eles: EinScan Pro (EP), EinScan Pro 2X Plus (EP +) (Shining 3D Tech. Co., Ltd. Hangzhou, China), iPhone X (IPX) (Apple Store, Cupertino, CA, EUA) e Planmeca ProMax 3D Mid (PM) (Planmeca EUA, Inc. IL, EUA). Para comparar as digitações realizadas por estes quatro equipamentos, foi utilizado o compasso de Vernier. Diante de seus resultados descobertos, os autores relataram que é recomendável usar o EinScan Pro 2X Plus para a digitalização de rosto para design baseado em rosto e outros fins de digitalização.

Lee $\mathrm{KH}$, et al. (2015) realizou um ajuste marginal de coroas sem metal feitas por três sistemas diferentes de projeto / fabricação auxiliado por computador CAD/CAM. Solaberrieta E, et al. (2015) em uma das suas pesquisas, analisou um articulador virtual odontológico para simular e analisar os movimentos mandibulares, e a realização de uma prótese total utilizando o CAD/CAM, e ainda mais, o autor relata que alguns dos dentistas que já oferecem essas tecnologias mais avançadas em suas clínicas dentais, onde estão passando a chamá-las: "odontologia de visita única".

Magano F, et al. (2019) apresentou um método digital que combina digitalização intraoral e facial para a fabricação de design assistido por computador / fabricação assistida por computador (CAD / CAM) de barras suportadas por implantes para overdentures maxilares, ou seja, em seu estudo clínico, realizou a integração dos exames intraoral e facial que permitiu restaurar com sucesso 15 overdentures maxilares totalmente edêntulos, suportadas por 4 implantes e uma barra CAD/CAM de poliéter-éter-cetona (PEEK). Assim como, em outro estudo, o mesmo autor apresentou um novo protocolo totalmente digital para o projeto e fabricação de coroas de zircônia translúcida monolítica suportadas por implantes cimentadas em abutments híbridos personalizados.

Gan N, et al. (2016) comparou a exatidão (veracidade e precisão) das impressões digitais intraorais para maxilares superiores inteiros, incluindo as dentições completas e tecidos moles palatinos, bem como determinar o efeito de diferentes alturas da abóbada palatina ou largura do arco na exatidão de impressões digitais intraorais.

Como relatado anteriormente, o CAD/CAM pode ser utilizado em diversas áreas, inclusive na área cirúrgica, sendo que no estudo de Mehra M, et al. (2016), demonstrou que o advento da tecnologia CAD/CAM, na utilização de cirurgia, pode ser planejado virtualmente para facilitar osteotomias precisas, levando a melhores resultados funcionais em reconstruções maxilofaciais, sendo que Gan N, et al. (2016) reforçou em seu estudo, que o scaneamento intraoral na maxila inteira, incluindo dentições completas e tecidos moles palatinos são parcialmente precisas, sendo que a largura do arco pode influenciar na precisão das impressões digitais intraorais, enquanto a altura da abóbada palatina pode não ter efeito na precisão das impressões digitais intraorais, e também são mais precisas para tecidos moles, e um pouco menos precisa para dentição completa.

Para auxiliar o cirurgião dentista, além das tecnologias apresentadas, Haenggi R, et al. (2016) demonstrou em seu estudo, uma ferramenta que permite aproximar o design digital do sorriso dos pacientes, onde os cirurgiões dentistas podem criar o seu próprio Smile designs de seus pacientes gratuitamente pelo Power Point, facilitando no diagnóstico de seus pacientes, aumentado ainda mais a eficiência clínica associada ao CAD/CAM. Com o uso CAD/CAM diminuiu o risco de contaminações, e para garantir a segurança contra contaminações, Barenchi L, et al. (2019) demonstrou no seu estudo, a eficiência da tecnologia para evitar a infecção nas clínicas odontológicas, pois interrompe ou reduz a infecção cruzada durante as etapas de impressão e fabricação. 


\section{CONSIDERAÇÕES FINAIS}

Diante do exposto, reconhecemos que a evolução e a modernidade dentro das clínicas odontológicas vêm em crescente aumento, havendo a necessidade dos cirurgiões dentistas se adequarem as tecnologias que estão sendo apresentadas no mercado. Com as tecnologias em crescente aumento, o cirurgião dentista que se enquadrar as tendências tecnológicas, poderão ter um diferencial competitivo no mercado, visto que os pacientes estão acompanhando a modernidade e buscam procedimentos que visam a eficiência e agilidade do profissional. Verificou-se que o uso do CAD/CAM traz vários benéficos ao paciente e principalmente ao cirurgião dentista, em que os mesmos, influenciam em ganho de eficiência e tempo de trabalho em suas clínicas, aumentando a qualidade e precisão do seu trabalho, assim como o tempo de espera do paciente ao seu tratamento. Os tratamentos propostos com o uso do CAD/CAM podem variar, desde a produção de coroas unitárias, facetas, prótese sobre implante, implantes dentários e até a realização de guias cirúrgicos.

\section{REFERÊNCIAS}

1. AMORNVIT P, SANOHKAN S. The Accuracy of Digital Face Scans Obtained from 3D Scanners: An In Vitro Study. International Journal of Environmental Research and Public Health, 2019; 16(24): 5061.

2. BARENGHI L, et al. Innovation by Computer-Aided Design/Computer-Aided Manufacturing Technology: A Look at Infection Prevention in Dental Settings. BioMed research international, 2019; 2019.

3. CUERVO JM. CAD-CAM en prótesis total. Reporte de caso. Universitas Odontológica, 2018; $37(78): 3$.

4. DEL CURTO F, et al. CAD/CAM-based chairside restorative technique with composite resin for full-mouth adhesive rehabilitation of excessively worn dentition. The international journal of esthetic dentistry, 2018; 13(1): 50.

5. GAN N, et al. Accuracy of intraoral digital impressions for whole upper jaws, including full dentitions and palatal soft tissues. PLoS One, 2016; 11(7): e0158800.

6. HAENGGI R, et al. Diseño de la sonrisa en PowerPoint. Rev. Asoc. Odontol. Argent, 2017; 28-32.

7. HASSAN B, et al. A digital approach integrating facial scanning in a CAD-CAM workflow for complete-mouth implantsupported rehabilitation of patients with edentulism: a pilot clinical study. The Journal of prosthetic dentistry, 2017a; 117(4): 486-492.

8. HASSAN B, et al. Integrating 3D facial scanning in a digital workflow to CAD/CAM design and fabricate complete dentures for immediate total mouth rehabilitation. The journal of advanced prosthodontics, 2017b; 9(5): 381-386.

9. LEE JD, et al. Integrated Digital and Conventional Treatment Workflow in Guided Complete Mouth Implant Rehabilitation: A Clinical Case Report. Dentistry Journal, 2019b; 7(4): 100.

10. LEE JH, et al. Contemporary full-mouth rehabilitation using a digital smile design in combination with conventional and computer-aided design/manufacturing restorative materials in a patient with bruxism: A case report. Medicine, 2019a; 98(48).

11. LEE KH, et al. Effects of computer-aided manufacturing technology on precision of clinical metal-free restorations. BioMed research international, 2015; 2015.

12. LESAGE BP. CAD/CAM: Applications for transitional bonding to restore occlusal vertical dimension. Journal of Esthetic and Restorative Dentistry, 2020; 32(2): 132-140.

13. MANGANO F, et al. A novel full-digital protocol (SCAN-PLAN-MAKE-DONE®) for the design and fabrication of implant-supported monolithic translucent zirconia crowns cemented on customized hybrid abutments: a retrospective clinical study on 25 patients. International Journal of Environmental Research and Public Health, 2019; $16(3): 3$.

14. MANGANO F, et al. Combining intraoral and face scans for the design and fabrication of computer-assisted design/computer-assisted manufacturing (cad/cam) polyether-ether-ketone (peek) implant-supported bars for maxillary overdentures. Scanning, 2019; 2019.

15. MEHRA M, et al. Mandibular fibular graft reconstruction with CAD/CAM technology: A clinical report and literature review. The Journal of prosthetic dentistry, 2016; 115(1): 123-128.

16. PYO SW, et al. Ceramic Materials and Technologies Applied to Digital Works in Implant-Supported Restorative Dentistry. Materials, 2020; 13(8): 1964.

17. ROMERO L, et al. New design for rapid prototyping of digital master casts for multiple dental implant restorations. PloS one, 2015; 10(12): e0145253.

18. SARATTI CM, et al. Full-mouth rehabilitation of a severe tooth wear case: a digital, esthetic and functional approach. The International Journal of Esthetic Dentistry, 2020; 15(3): 242-262.

19. SELZ C, et al. Full-mouth rehabilitation with monolithic CAD/CAM-fabricated hybrid and all-ceramic materials: A case report and 3-year follow up. Quintessence Int, 2016; 47(2): 115-21.

20. SOLABERRIETA E, et al. Virtual prouction of dental prostheses using a dental virtual articulator. International Journal on Interactive Design and Manufacturing (IJIDeM), 2015; 9(1): 19-30.

21. SUN YC, et al. Research and development of digital design and fabrication of complete denture. Zhonghua kou qiang yi xue za zhi= Zhonghua kouqiang yixue zazhi= Chinese journal of stomatology, 2018; 53(1): 60-65. 